\title{
Baja adherencia al régimen de hemodiálisis en pacientes con enfermedad crónica renal en un hospital de referencia del Ministerio de Salud en Perú
}

\author{
Low adherence to hemodialysis regime in patients with chronic renal \\ disease in a Peruvian Ministry of Health reference hospital
}

\begin{abstract}
Percy Herrera-Añazco ${ }^{1,4}$, Melissa Palacios-Guillen ${ }^{2,6}$, Edward Mezones-Holguin ${ }^{3,5}$, Adrián V. Hernández ${ }^{3,5}$, David Chipayo-Gonzales ${ }^{1}$

${ }^{1}$ Hospital Nacional Dos de Mayo, Lima, Perú.

${ }^{2}$ Hospital Daniel Alcides Carrión, Callao, Perú.

${ }^{3}$ Unidad de Análisis y Generación de Evidencias en Salud Pública (UNAGESP), Instituto Nacional de Salud, Lima, Perú.

${ }^{4}$ Universidad Nacional de Piura, Perú.

${ }^{5}$ Universidad Peruana de Ciencias Aplicadas, Perú.

${ }^{6}$ Universidad Nacional Mayor de San Marcos, Perú.
\end{abstract}

\begin{abstract}
Resumen
Introducción: La ausencia a una sesión mensual en un esquema de diálisis convencional puede incrementar la mortalidad en $30 \%$. Objetivos: Describir la frecuencia y la percepción de las causas de falta de adherencia a diálisis en una población prevalente de un hospital público de referencia nacional en Perú. Diseño: Estudio descriptivo. Institución: Servicio de Nefrología, Hospital Nacional 2 de Mayo, Lima, Perú. Participantes: Pacientes con más de un año en diálisis Intervenciones: Se determinó el número de faltas y se aplicó un cuestionario para describir su percepción respecto a las causas de las faltas, validado por juicio de expertos. Principales medidas de resultados: Baja adherencia a diálisis definida como: pacientes con más de una falta al mes o más de 12 faltas, entre julio de 2012 y julio de 2013. Resultados: Se incluyó 54 pacientes, 27 eran varones, con una edad y tiempo de diálisis promedio de $57 \pm 16,4$ años y 40,6 $\pm 11,5$ meses, respectivamente; $7 / 54$ pacientes tenían educación superior. Hubo 504 faltas (5,45\%). El segundo día de la programación semanal fue el día con mayor frecuencia de faltas (292), seguido del tercer dia (145); $13 / 54$ tuvieron baja adherencia. Las principales causas reportadas fueron: una residencia alejada (6/13), la sensación de bienestar (6/13), el contar con escasos recursos económicos para solventar el traslado (5/13). Conclusiones: Uno de cada cuatro pacientes tuvo baja adherencia. El residir lejos o que se sintiera bien fueron las principales causas de la baja adherencia.
\end{abstract}

Palabras clave: Diálisis, insuficiencia renal, pacientes desistentes del tratamiento, Perú.

\section{Abstract}

Background: Failing to attend a monthly session within a scheme of conventional dialysis may increase mortality by $30 \%$. Objectives: To describe the frequency and perceived causes of non-adherence to dialysis in a Peruvian national reference public hospital. Design: Descriptive study. Setting: Nephrology department, Hospital Nacional 2 de Mayo, Lima, Peru. Participants: Patients with more than one year on dialysis. Interventions: The number of absences to appointed sessions was determined and a validated questionnaire was used to describe perceptions regarding the causes of absences. Main outcomes measures: Low adherence to dialysis defined as patients with more than one absence per month or more than 12 absences between July 2012 and July 2013. Results: The study included 54 patients, of which 27 were male. Average age was $57 \pm 16.4$ years and average time on dialysis was $40.6 \pm 11.5$ months. Only 7 patients had higher education. There were 504 absences (5.45\%). The second day of the weekly schedule was the day with more absences (292), followed by the third day (145). Overall 13 patients showed low adherence. Main causes of absence reported included a remote residence (6/13), feeling good (6/13), and insufficient financial resources to cover transportation costs (5/13). Conclusions: A quarter of patients had low adherence, and main factors were distance to health facility and wellbeing self-perception.

Keywords: Dialysis, renal insufficiency, patient dropouts, Peru.

An Fac med. 2014;75(4):323-6 / doi: http://dx.doi.org/10.15381/anales.v75i4.10848 


\section{INTRODUCCIÓN}

La hemodiálisis (HD) convencional es el tipo de diálisis más usada en los pacientes con enfermedad renal crónica en estadio 5 (ERC5) ${ }^{(1)}$, la misma que requiere una frecuencia y una duración adecuada para conseguir el objetivo de mejorar la calidad de vida de estos pacientes ${ }^{(2)}$.

La falta de adherencia al régimen de diálisis traducida en menos sesiones que las requeridas, se asocia a mayor mortalidad ${ }^{(3,4)}$, de tal forma que la ausencia a una sesión mensual en un régimen convencional de tres sesiones por semana incrementa la probabilidad de morir hasta en $30 \%{ }^{(4)}$.

Las causas estudiadas que explican esta falta de adherencia incluyen tanto factores sociodemográficos, psicológicos como logísticos, muchas de ellas potencialmente modificables ${ }^{(6-8)}$.

En Perú, existen problemas de cobertura de la terapia de diálisis en los pacientes con ERC5 en el Ministerio de Salud (MINSA) ${ }^{(8)}$, por lo que presumimos que la falta de adherencia podría ser alta, no existiendo estudios que evalúen este problema, así como las potenciales causas de la misma. Nuestro objetivo fue describir la frecuencia y la percepción de causas de falta de adherencia a $\mathrm{HD}$ en una población prevalente de un hospital público de referencia nacional en Perú.

\section{MÉTODOS}

Llevamos a cabo un estudio descriptivo de serie de casos en los pacientes mayores de 18 años con ERC5 que tenían más de un año en HD en el Servicio de Nefrología del Hospital Nacional 2 de Mayo en Lima, Perú. Excluimos a los pacientes que se rehusaron a participar o que al momento de la evaluación no pudieron dar datos sobre lo preguntado (por demencia senil, alteración de nivel de conciencia, entre otros).

Se recogieron datos demográficos y clínicos y se definió como baja adhe- rencia a aquellos pacientes con más de una falta al mes o más de 12 faltas en el último año ${ }^{(4)}$. Los pacientes fueron categorizados a partir del registro de enfermería de HD de julio de 2012 a julio de 2013.

En los pacientes con baja adherencia aplicamos un cuestionario heteroaplicado para describir su percepción respecto a las causas de las faltas. El cuestionario fue validado por juicio de expertos en base a entrevistas a profundidad y estudios previos ${ }^{(6-8)}$.

Para la descripción de las variables categóricas utilizamos frecuencias absolutas y relativas y para las variables continuas usamos media y desviación estándar.

\section{RESULTADOS}

Incluimos 54 pacientes, la mitad eran varones, con una edad promedio de 57 $\pm 16,4$ años y un tiempo de diálisis promedio de 40,6 $\pm 11,5$ meses.

La diabetes mellitus fue la causa más frecuente de ERC en 27 pacientes, seguida de la glomerulonefritis crónica en 11 casos. La uropatía obstructiva, la causa no filiada y otras causas tuvieron 4 pacientes cada una. La hipertensión arterial fue la causa de ERC en 3 pacientes y la poliquistosis renal en un paciente.

Treinta y tres pacientes tenían educación secundaria, 12 pacientes solo educación primaria, 7 pacientes educación superior y 3 pacientes eran analfabetos.
La relación de sesiones de diálisis y el número de faltas se muestran en la tabla 1.

Las principales causas de baja adherencia fueron: vivir en una residencia alejada del hospital (6/13); se siente bien y cree que no necesita diálisis (6/13); no cuenta con recursos económicos para el traslado (5/13); adolece de falta de compañía para el traslado (3/13); y, quiere ahorrar dinero del seguro de salud (1/13).

\section{DISCUSIÓN}

Nuestros principales hallazgos muestran una alta frecuencia de falta de adherencia a las sesiones de HD, siendo la mayoría de ellas secundarias a factores socioeconómicos.

Los estudios que han comunicado una falta de adherencia muestran frecuencias variadas, llegando a ser tan altas como 32,3\% en algunos reportes. Sin embargo, esta frecuencia varía de país en país ${ }^{(9)}$. En el estudio multinacional The Dialysis Outcomes and Practice Patterns Study (DOPPS) se encontró que $3,8 \%$ del total de los pacientes estudiados había tenido más de 1 falta al mes; no obstante, esta había sido 7,9\% en Estados Unidos y 0,6\% tanto en Japón como en los países europeos ${ }^{(4)}$. Por otro lado, en estudios específicos en Europa también se comunica diferentes frecuencias. Así, se ha informado que esta es $0,3 \%$ en Francia, 0,9\% en Alemania, 8,8\% en Italia, 6,6\% en España y $12,6 \%$ en Inglaterra ${ }^{(9)}$, porcentajes mucho menores que en nuestro estu-

Tabla 1. Falta de adherencia a diálisis.

$\begin{array}{cc}\text { Variables } & \text { N }=54 \text { pacientes } \\ \text { Número total de sesiones de diálisis } & 9234 \\ \text { Número de faltas a sesiones de hemodiálisis } & 504 \\ \text { Faltas el primer día de la semana } & 67 \\ \text { Faltas el segundo día de la semana } & 292 \\ \text { Faltas el tercer día de la semana } & 145 \\ \text { Número de pacientes con baja adherencia } & 13\end{array}$


dio, donde el $24 \%$ de los pacientes tuvo baja adherencia a diálisis.

En América Latina, una comunicación colombiana mostró que se había incumplido $1,4 \%$ del total de sesiones de diálisis estudiadas ${ }^{(10)}$, cantidad menor al cerca de $6 \%$ del total de las sesiones evaluadas en nuestro trabajo.

La falta de adherencia a diálisis se ha asociado a hiperkalemia, hiperfosfatemia y baja probabilidad de trasplante renal en pacientes menores de 65 años ${ }^{(11)}$, pero sobre todo a alta mortalidad en pacientes en HD ${ }^{(3,4)}$, por lo que el conocimiento de los factores asociados es de importancia para identificar cuáles son los potencialmente modificables. De esta manera, la falta de adherencia se ha asociado a pacientes de raza negra, fumadores, y al nivel de escolaridad ${ }^{(6,12)}$. Por otro lado, al estudiarse las razones de la falta de adherencia, se ha hallado a las obligaciones diarias en la casa y problemas en el transporte ${ }^{(12)}$. Otros factores identificados son la confianza en el equipo médico, las redes de apoyo a los pacientes, la aceptación de la enfermedad, los efectos secundarios de la terapéutica, la falta de acceso a los medicamentos, el tratamiento largo, el esquema terapéutico complejo y la ausencia de síntomas ${ }^{(6,7)}$. Afortunadamente, se ha encontrado que la concientización de los pacientes sobre las consecuencias de la inasistencia a las sesiones de diálisis podría potencialmente revertir este problema ${ }^{(10)}$, lo que demuestra la necesidad de la capacitación continua tanto a los pacientes como al personal encargado de la atención en diálisis.

En nuestro trabajo llama la atención que, además de la supuesta ausencia de síntomas como principal causa de esta falta de adherencia, el residir lejos y el carecer de recursos económicos para el traslado fueran también factores importantes. Se ha descrito que la cobertura de diálisis en el MINSA es deficiente, por lo que muchos pacientes se ven con problemas para encontrar cupos en los hospitales con centros de diálisis operativos ${ }^{(10)}$, de tal forma que en nuestro hospital solo $20 \%$ de nuestros pacientes pertenece a nuestra red asistencial ${ }^{(13)}$, lo que obliga a cubrir mayores distancias para llegar a sus sesiones de diálisis y que se ha descrito como causa de mayor mortalidad y peor calidad de vida entre los pacientes en diálisis ${ }^{(14)}$. Por otro lado, esta falta de recursos económicos para el traslado así como su nivel educativo demuestra el tipo de pacientes que tenemos en los hospitales del MINSA y es de importancia, ya que al igual que los factores clínicos, la falta de recursos económicos se ha asociado a baja calidad de vida en pacientes en diálisis ${ }^{(15)}$.

Si bien se ha sugerido que una menor frecuencia de diálisis podría ser posible en pacientes que inician diálisis y tienen una adecuada función renal residual ${ }^{(16)}$, o pacientes que inician los cuidados paliativos cerca al final de su vida ${ }^{(17)}$, se ha comunicado que un mayor periodo de tiempo interdialítico en la hemodiálisis convencional, sobre todo en el periodo previo al primer día de la semana, está relacionado a mayor mortalidad ${ }^{(18)}$. Por eso llama la atención que el segundo día de mayor inasistencia fuera el último día de la semana, con el consiguiente riesgo de que nuestros pacientes tengan cerca de cuatro días sin diálisis.

Nuestro trabajo es unicéntrico, por lo que no es posible generalizar los hallazgos a otros centros del MINSA o a hospitales de EsSalud. Sin embargo, el hospital nacional 2 de Mayo es el hospital de Lima con la mayor cantidad de pacientes en diálisis crónica, por lo que nuestros hallazgos bien pueden ser un reflejo de lo que pasa en el resto de hospitales. Por otro lado, dado lo pequeño de la muestra no es posible inferir causalidades. De nuestro conocimiento, es el primer trabajo que se realiza al respecto en nuestro país.

En conclusión, cerca de uno de cada cuatro pacientes tiene baja adherencia. El residir lejos o que se sienta bien son las principales causas de la falta de adherencia, por lo que es necesario reforzar la educación continua en nuestros pacientes sobre las consecuencias de la falta de adherencia a diálisis, así como invocar a las autoridades del MINSA a diseñar un plan nacional de terapias de reemplazo renal con el fin de mejorar las redes de atención de los pacientes en diálisis.

\section{REFERENCIAS BIBLIOGRÁFICAS}

1. Karopadi AN, Mason G, Rettore E, Ronco C. Cost of peritoneal dialysis and haemodialysis across the world. Nephrol Dial Transplant. 2013 Oct;28(10):2553-69. doi: 10.1093/ndt/gft214.

2. ERA EDTA Guidelines: European best practice guidelines for hemodialysis (part 1). Nephrol Dial Transplant. 2002;17(suppl 7):25-31.

3. Saran R, Bragg-Gresham JL, Rayner HC, Goodkin DA, Keen ML, et al Nonadherence in hemodialysis: Associations with mortality, hospitalization, and practice patterns in the DOPPS. Kidney Int. 2003 Jul;64(1):254-62.

4. Saran R, Bragg-Gresham JL, Rayner HC, Goodkin DA, Keen ML. Noncompliance in hemodialysis: predictors and survival analysis. Kidney Int. 2003 Jul;64(1):254-62

5. Gordon EJ, Leon JB, Sehgal AR. Why are hemodialysis treatments shortened and skipped? Development of a taxonomy and relationship to patient subgroups. Nephrol Nurs J. 2003 Apr;30(2):209-17

6. Maldaner C, Beuter M, Brondani C, Budó M, Pauletto M. Fatores que influenciam a adesão ao tratamento na doença crónica. Rev Gaúcha Enferm. 2008 dez;29(4):647-53.

7. Madeiro A, Lopez Carrillo P, Melo I, Ribeiro A, et al. Adesão de portadores de insuficiência renal crônica ao tratamento de hemodiálise. Acta Paul Enferm. 2010;23(4):546-51.

8. Herrera-Anazco P, Mezones-Holguín E, Hernandez AV. Global kidney disease. Lancet. 2013 Oct 12;382(9900):1243-4. doi: 10.1016/S0140-6736(13)62087-5

9. Denhaerynck K, Manhaeve D, Dobbels F, Garzoni $D$, Nolte $C$, et al. Prevalence and consequences of nonadherence to hemodialysis regimens. Am J Crit Care. 2007 May;16(3):222-35.

10. Acosta C, Sepúlveda G, Ibañez E, Flores C, Herran $\mathrm{M}$ y col. Adherencia de los pacientes con insuficiencia renal crónica a las sesiones de hemodiálisis del hospital militar centra, Bogotá, DC 2006 - 2007. Rev Colomb Enferm. 2008;3(3):61-7.

11. Unruh ML, Evans IV, Fink NE, Powe NR, Meyer KB. Skipped treatments, markers of nutritional nonadherence, and survival among incident hemodialysis patients. Am J Kidney Dis. 2005 Dec;46(6):1107-16.

12. Gordon EJ, Leon JB, Sehgal AR. Why are hemodialysis treatments shortened and skipped? Development of a taxonomy and relationship to patient subgroups. Nephrol Nurs J. 2003 Apr;30(2):209-17.

13. Herrera P, Palacios M, Chipayo D, Silveira M. Mortalidad durante la primera hospitalización en una población que inicia diálisis crónica en un hospital general. An Fac med. 2013;74(3):199-202. 
14. Moist LM, Bragg-Gresham JL, Pisoni RL, Saran $\mathrm{R}$, Akiba $\mathrm{T}$, et al. Travel time to dialysis as a predictor of health-related quality of life, adherence, and mortality: the Dialysis Outcomes and Practice Patterns Study (DOPPS). Am J Kidney Dis. 2008 Apr;51(4):641-50.

15. Marinovich S, Laborato C, Rosa G, Bisigniano L, Fernandez $V$ y col. La falta de ingresos económicos se asocia a menor supervivencia en hemodiálisis crónica. Nefrologia. 2012;32(1):79-88.

16. Rhee CM, Unruh M, Chen J, Kovesdy CP, Zager $P$, Kalantar-Zadeh K. Infrequent dialysis: a new paradigm for hemodialysis initiation. Semin Dial. 2013 Nov-Dec;26(6):720-7.
17. Kidney Care National End of Life Care. Programme. End of Life Care in Advanced Kidney Disease: A Framework for Implementation. 2009. Disponible en: http://www.ncpc.org.uk/sites/default/files/EndOfLifeCarelnAdvancedKidneyDisease.pdf

18. Foley RN, Gilbertson DT, Murray T, Collins AJ. Long interdialytic interval and mortality among patients receiving hemodialysis. N Engl J Med. 2011 Sep 22;365(12):1099-107.

Artículo recibido el 1 de abril de 2014 y aceptado para publicación el 19 de abril de 2014.
Conflictos de interés:

Los autores declaran no existir conflictos de interés.

Financiamiento:

Propio de los autores.

Contacto:

Dr. Percy Herrera Añazco

Dirección: Olavegoya 1879 Dpto 701 Jesús María,

Lima, Perú

Correo electrónico: silamud@gmail.com 